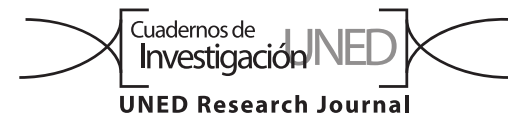

\title{
Impacto y oportunidades de biorrefinería de los desechos agrícolas del cultivo de piña (Ananas comosus) en Costa Rica
}

\author{
Rodolfo A. Hernández-Chaverri' \& Lilia Arely Prado Barragán² \\ 1. Cátedra de Ciencias Químicas para Ingenierías, Unidad de Investigaciones Bioenergéticas y Ambientales, Universidad Estatal a Distancia, \\ Costa Rica; rohernandez@uned.ac.cr \\ 2. Departamento de Biotecnología, Planta Piloto 4, Universidad Autónoma Metropolitana, Iztapalapa, México.
}

Recibido 03-IV-2018 • Corregido 04-VI-2018 • Aceptado 14-VI-2018

\begin{abstract}
Impact and biorefinery opportunities for agricultural waste of the cultivation of pineapple (Ananas comosus) in Costa Rica. Costa Rica is the world's leading exporter of fresh pineapple (Ananas comosus); associated with the pineapple's agricultural production, comes the issue of agricultural waste and the interest in pineapple stubble as the main discarding material. We estimated the biomass that is generated from pineapple stubble, the costs associated with field management and identified the current treatment that this waste is given; as well as an initial analysis of strengths, weaknesses, opportunities and threats. It is estimated that the biomass of pineapple stubble for $43000 \mathrm{Ha}$ of current crop is $4,282 \times 10^{6}$ tons/year on a wet basis $(640$ thousand ton/year dry base). The associated costs for management in the pineapple stubble field range between US\$ 1000 and US\$2 500 by hectare, depending on the type of management; dry (chemical drying with Paraquat) or green handling (crushing of the green plant) and its incorporation into the ground. Within the economy of export of agricultural products is the second largest, behind the banana, since more than US\$900 million are exposed annually (2017), which makes it possible to add another biomass, such as banana, coffee, oil palm and forest products, to the development of a bioenergy and bio manufacturing industry. It is important that the biorefinery processes are integrated into industrial ecology models or circular economy, to minimize the production of greenhouse gases and allow food security in the regions, with the aim of achieving the objectives of sustainable development.
\end{abstract}

Key words: biorefinery, stubble of pineapple, biomass, greenhouse gases.
RESUMEN: Costa Rica es el principal exportador mundial de piña fresca (Ananas comosus); asociado a la producción agrícola de la piña, viene el tema de los residuos agrícolas y el interés en el rastrojo de piña como principal material de descarte. Hicimos una estimación de la biomasa que se genera de rastrojo de piña, los costos asociados con el manejo en campo e identificamos el tratamiento actual que se da a este residuo; además de un análisis inicial de fortalezas, debilidades, oportunidades y amenazas. Estimamos que la biomasa de rastrojo de piña para $43000 \mathrm{Ha}$ actuales de cultivo es de 4,282 × 10 $10^{6}$ ton/año en base húmeda (640 mil ton/año base seca). Los costos asociados para la gestión en el campo del rastrojo de piña oscilan entre US\$ 1000 y US\$ 2500 por hectárea, dependiendo del tipo de manejo; en seco (secado químico con Paraquat) o manejo en verde (trituración de la planta verde) y su incorporación al terreno. Dentro de la economía de exportación de productos agrícolas es la segunda mayor, detrás del banano, ya que se exportan más de US\$ 900 millones al año (2017), lo cual hace que existan oportunidades de mejora en el manejo y aprovechamiento de esta biomasa, y se deba valorar el estudio a mayor profundidad del desarrollo de un proceso de biorrefinería para la obtención de productos de mayor valor agregado como biocombustibles, energía, biomoléculas, entre otros. Es importante que los procesos de biorrefinería que se propongan se integren en modelos de ecología industrial o economía circular, para minimizar la producción de gases de efecto invernadero y permitir la seguridad alimentaria en las regiones, con la finalidad de alcanzar los objetivos del desarrollo sostenible.

Palabras clave: biorrefinería, rastrojo de piña, biomasa, gases de efecto invernadero.
Costa Rica es el principal exportador mundial de piña fresca (Ananas comosus) como lo indican Garita (2014) y De Pablo Valenciano, Tassile y Giacinti Battistuzzi (2017). Asociado a la producción agrícola de la piña, viene el tema de los residuos agrícolas y el interés en el rastrojo de piña como principal material de descarte. No hay con certeza una estimación exacta de la cantidad de rastrojo que se puede generar por hectárea cultivada, ya que depende de la densidad de la siembra entre otros. Plantas de mayor peso tendrán mayor biomasa, una primera estimación la hace Quesada-Solís, Alvarado Aguilar, Sibaja Ballestero y Vega Baudrit (2005) en 300 toneladas métricas por hectárea en fresco (material húmedo).

Garita (2014), indica que en un periodo relativamente corto se han presentado situaciones que propiciaron el cultivo de piña en Costa Rica como lo son: 
- La instalación de la transnacional Del Monte en el país, alrededor de 1977. Con Del Monte se desarrolla el cultivo la piña y expansión de este en diferentes zonas del país, inicialmente en la zona sur (Buenos Aires de Puntarenas, Costa Rica).

- La sustitución de la variedad Smooth Cayena por la variedad Ilamada "Champaka F-153", este es uno de los primeros cambios a una variedad de mayor tamaño y dulzor.

- El desarrollo y siembra a gran escala de una nueva variedad M2 para el año 1996; esta es la piña que se siembra y exporta con la mejor adaptación a los suelos de las diferentes zonas de cultivo, incluso mejorando en sabor a la que se cultiva en Hawái. Esta última sin duda revolucionó la exportación de piña a nivel mundial por sus características organolépticas.

La Cámara Nacional de Productores y Exportadores de Piña de Costa Rica (CANAPEP) indican que actualmente existen cerca de $43000 \mathrm{Ha}$ dedicadas al cultivo de piña en Costa Rica, a todo lo largo y ancho de 16 cantones diferentes. Las áreas de cultivo de este producto se concentran en 4 regiones: zona Norte con $20210 \mathrm{Ha}$, lo que equivalente al $47 \%$ del área cultivada; zona atlántica que dispone de $13330 \mathrm{Ha}$ y representa el $31 \%$, a su vez, la región Pacífico Sur destina $9460 \mathrm{Ha}$, lo cual constituye el $22 \%$ del área cultivada y en Pacífico Central que inicia la actividad. Se reportan cerca de 550 productores, y se estima un empleo directo de 30000 personas con la actividad del cultivo de piña (Fig. 1; CANAPEP, 2017).

Maglianesi (2013) indica que, alrededor del cultivo de piña se ha desarrollado un sistema productivo dependiente de los insumos químicos, en los que se contempla el uso de herbicidas, fungicidas, nematicidas, insecticidas, abonos y compuestos químicos que inducen la floración de la planta y regulan la cosecha. Para Salazar (2008) la cantidad de insumos químicos utilizados en la producción de la piña supera por mucho la cantidad utilizada en la producción bananera, por la particularidad del ciclo productivo del fruto, el cual se debe acelerar en función de la demanda mundial. Por otra parte, como resultado de la expansión de la actividad piñera se instalaron complejos industriales para la recolección y empaque del producto, cambiando drásticamente el paisaje en varios sitios del país.

En su investigación Navarro y Gómez (2015), indican que la fragmentación del hábitat producida por el cultivo de piña, sumado a la fuerte presión por cacería podrían ser las causas de la disminución de poblaciones silvestres que se encuentran confinadas a sitios específicos debido a la baja conectividad. De este modo, la producción de piña aumenta el peligro de que las especies silvestres, principalmente de mamíferos, desaparezcan en zonas habituales a su distribución histórica. Es así, que el XXI Programa Estado de la Nación (2015) indica que se entra en una situación de riesgo, entre la sostenibilidad económica de un monocultivo como la piña y la pérdida o disminución ecológica de especies, bosques y corredores biológicos. Esto a raíz del uso intensivo del suelo y de la aplicación de productos químicos en las diferentes etapas de la producción de piña. Los aumentos en el área de producción en los cultivos de exportación (piña, palma aceitera y banano tienen implicaciones ambientales serias para la sostenibilidad del ambiente. Esto por cuanto son monocultivos que se caracterizan por el uso

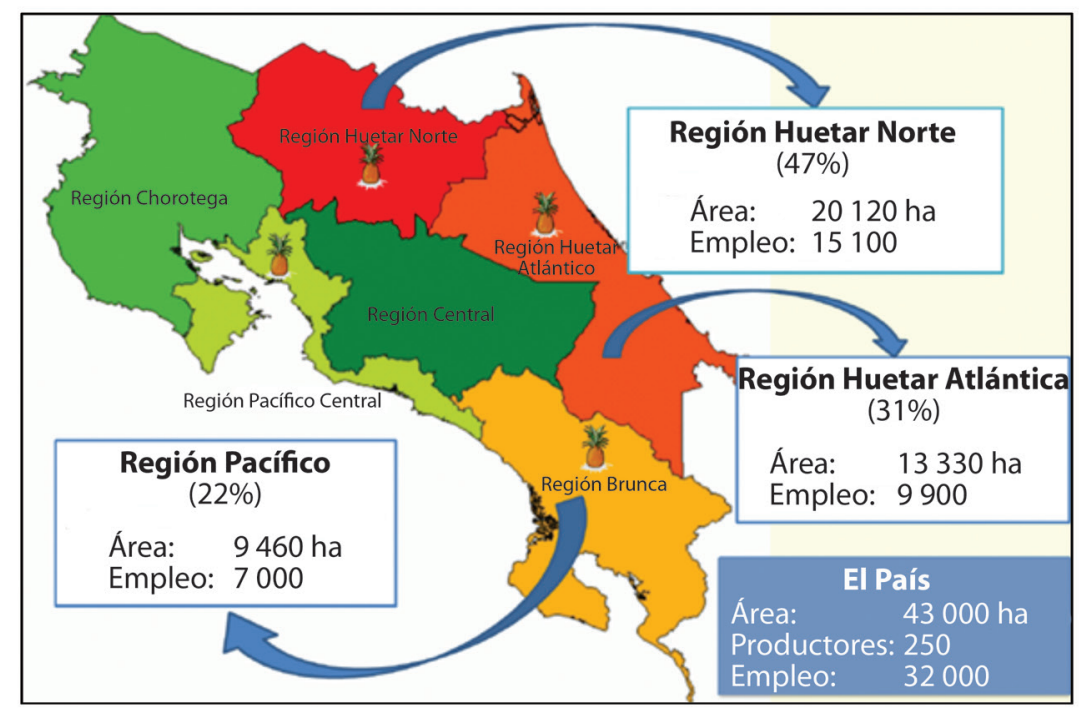

Fig. 1. Distribución de la siembra de piña en Costa Rica. Fuente (CANAPEP, 2017). 
intensivo de agroquímicos. Si bien se han dado iniciativas para la producción más amigable con el ambiente, en piña y banano, éstas no parecen ser financieramente viables, lo que representa un desincentivo.

Como bien se indica en el XXI Programa Estado de la Nación (2015), el área dedicada al cultivo de la piña creció quince veces, mientras que la cantidad de fincas decreció cerca de $62 \%$ en el periodo comprendido 1984 a 2014. Esto evidencia que el proceso de expansión de tierras dedicadas al cultivo de la piña ha ido en aumento. Lo anterior a expensas de la disminución de las fincas o personas dedicas al cultivo, dándose una concentración de las tierras de cultivo en manos de muy pocos. Esto coincide con el alto porcentaje de productores pequeños que han vendido o alquilado sus tierras a los grandes productores. El incremento en la producción de piña provoca a su vez un incremento de los desechos generados por la actividad. Así, resulta fundamental valorar el aprovechamiento de la biomasa del rastrojo de piña que representa el principal producto de desecho.

Una forma de aprovechamiento puede ser el uso del rastrojo de piña como materia prima para un proceso de biorrefinería (proceso donde se llevan a cabo transformaciones bioquímicas o termoquímicas de biomasa lignocelulósica). Dimian (2015), menciona que debe tenerse un enfoque basado en el aprovechamiento de los recursos y economía circular, lo que significa reciclar los productos usados y los residuos. La biomasa tiene la ventaja de ser el portador de energía natural del sol a la vida terrestre, por lo que su aprovechamiento implica el uso de energía renovable. La tecnología química moderna, en asociación con otras áreas del conocimiento como la agricultura y la agroindustria, tienen que resolver la desafiante tarea de reemplazar el procesamiento de hidrocarburos fósiles por la conversión de biomasa en productos similares o nuevos. La implementación de este modelo implica la oportunidad de generar tecnologías avanzadas para la minimización de la contaminación derivada de la actividad energética actual y el aprovechamiento de los recursos; así como, para reciclar los residuos de la agricultura, la industria o el de actividades domésticas. Nuevas "químicas verdes" tienen que ser inventadas y el diseño de procesos sostenibles tiene que ser generalizado.

Esta investigación tiene como objetivo establecer las implicaciones socio ambientales y económicas del cultivo de la piña con el fin de analizar alternativas para el manejo integral del rastrojo de piña desde una perspectiva de biorrefinería.

\section{MATERIALES Y MÉTODOS}

Zona de estudio: Se define como zona geográfica para este estudio, la zona norte de Costa Rica (Fig. 2., delimitada en rojo), la misma colinda al norte con Nicaragua, al este con las Ilanuras de la zona atlántica, al sur con las montañas de la cordillera de Tilarán y al oeste con los

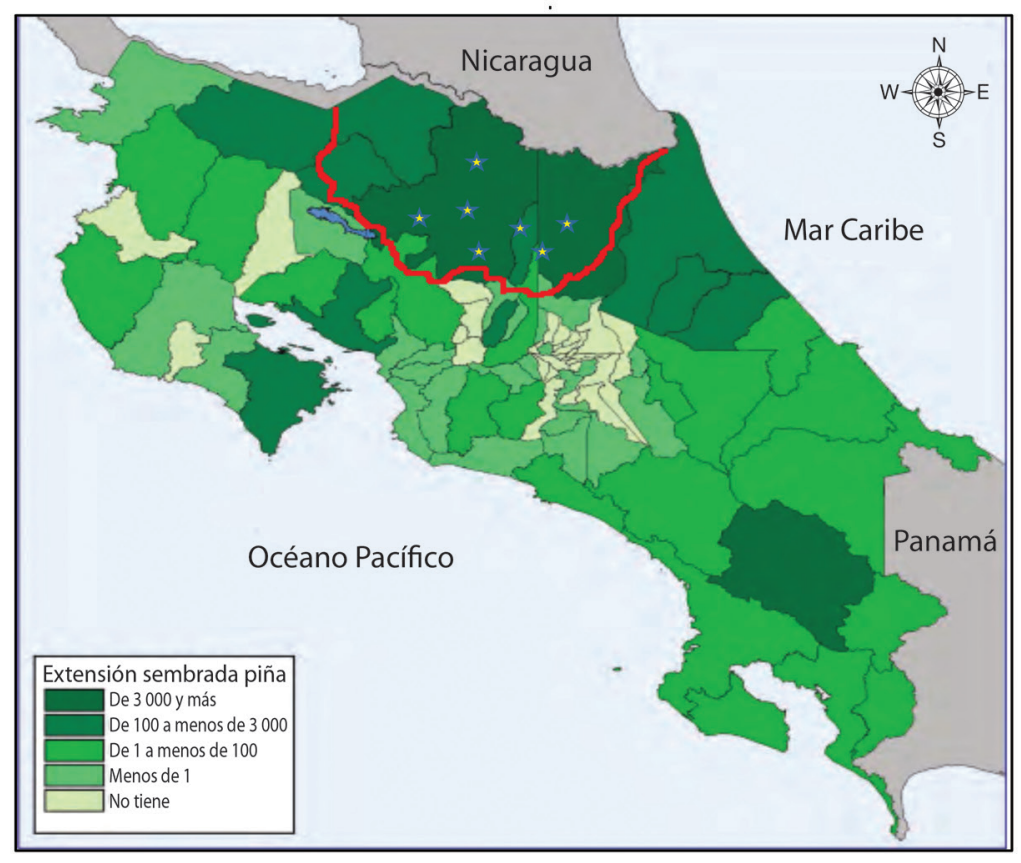

Fig. 2. Delimitación del área de estudio, a partir de mapa de densidad de siembra del cultivo de piña. Fuente: INEC, 2017. 
volcanes Tenorio y Miravalles. Se muestrean siete fincas que comprenden un área de siembra $4000 \mathrm{Ha}$, con una distribución homogénea sobre el área delimitada y representativa del cultivo de piña en Costa Rica.

Revisión de bases de datos: Se realizó una revisión de las estadísticas de exportación de la Promotora de Comercio Exterior (PROCOMER, 2018) para cultivos como la piña, banano, café; así como de las estadísticas del Instituto Nacional de Estadística y Censos (INEC, 2017) en relación al último censo agropecuario de 2014 de Costa Rica para identificar zonas cultivadas y los mayores monocultivos.

Entrevistas de campo: Se llevaron a cabo entrevistas a diferentes actores relacionados con el cultivo y control de la producción de piña mediante una encuesta no estructurada. La misma tuvo la finalidad de estimar parámetros como: relación área sembrada/área protegida, taza de recambio anual del cultivo, costos asociados a la preparación del terreno para siembra, manejo del rastrojo; además, de establecer que es lo que se define como rastrojo de piña para el productor y la posición del Sistema Fitosanitario del Estado (SFE).

\section{Muestreo y estimación de la biomasa en campo:}

Por otra parte, a partir de un muestreo aleatorio simple en finca en combinación con la técnica de cuadrante, se toman muestras de las plantas declaradas como rastrojo de piña para determinar su masa en húmedo (siete fincas, cuatro lotes por finca, entre 15 y 20 plantas por lote, en diferentes épocas del año). De manera empírica se realizó la estimación de la biomasa (rastrojo de piña) que se genera anualmente en el cultivo de la piña, este proceso consistió en tomar las muestras en cada finca, llevarlas al área de laboratorio, lavar las raíces y la planta en general, y dejar secar al ambiente por cuatro horas, para luego medir la masa con una balanza de resorte. La estimación de la biomasa anual se realizó aplicando la ecuación 1, la cual es parte del desarrollo de esta investigación.

$$
M R P H=A C \cdot R R C \cdot R V S \cdot D S \cdot M P I \cdot \frac{1}{1000}
$$

Donde:

MRPH: Masa de rastrojo de piña (fresco) húmedo (toneladas/año).

AC: $\quad$ Área de cultivo (hectáreas).

RRC: Razón de recambio del área de cultivo anual.
RVS: Razón de vida de las plantas sembradas.

DS: $\quad$ Densidad de siembra (plantas/hectárea).

MPI: Masa planta individual (kilogramos).

Matriz de fortalezas-oportunidades y debilidades-amenazas: Con los datos, anteriormente recolectados y/o generados se construyó una matriz de análisis de fortalezas-oportunidades y debilidades-amenazas. A partir de ésta se establecen una serie de estrategias vinculantes con el rastrojo de piña y un modelo teórico de un proceso de biorrefinería sobre el cultivo de piña.

Determinación de humedad y materia seca: Para la determinación de humedad y materia seca se sigue el procedimiento estándar del National Forage Testing Association (NFTA), referencia del método NFTA Method 2.1.4 (2006).

\section{ANÁLISIS}

A partir de las visitas a las fincas y conversaciones con productores y agentes del SFE, se establece que el rastrojo de piña en campo es aquel compuesto por la totalidad de la planta de piña (raíz, tallo, hijos secundarios y hojas), una vez que se descarta y se inicia un nuevo ciclo de siembra. Hay que destacar que de las plantas antes del descarte, se toman los brotes (hijos secundarios) para el reemplazo en un nuevo ciclo de siembra, muy ocasionalmente se utiliza la corona de la fruta como semilla (Fig. 3).

Datos económicos: A partir de los datos del Instituto Nacional de Estadísticas y Censos (INEC) en el VI Censo Agropecuario de 2014 (INEC, 2017) se muestra en la figura 4, como el cultivo de piña ocupa la séptima posición en área cultivada de monocultivos en Costa Rica, lo cual se ha alcanzado en menos de 30 años como cultivo intensivo. En especial si se observan las áreas de cultivo del café o banano que tienen más de 130 años de ser sembrados en Costa Rica.

En la figura 5 se comparan las exportaciones de tres productos agrícolas de exportación, el café, banano y piña de Costa Rica. Como se observa, en menos de 25 años el cultivo de piña alcanzó y rebasó al cultivo del café, y está muy cerca del cultivo del banano. Al comparar con la figura 3, en la que se observa que el área sembrada de piña es menor a los otros dos productos, el impacto de generación de divisas por hectárea es mucho mayor para la piña (al 2014, US\$3 300 para café por hectárea, US\$ 17500 para banano y US\$23 600 para piña, al dividir dólares exportados entre las hectáreas cultivadas de ese 

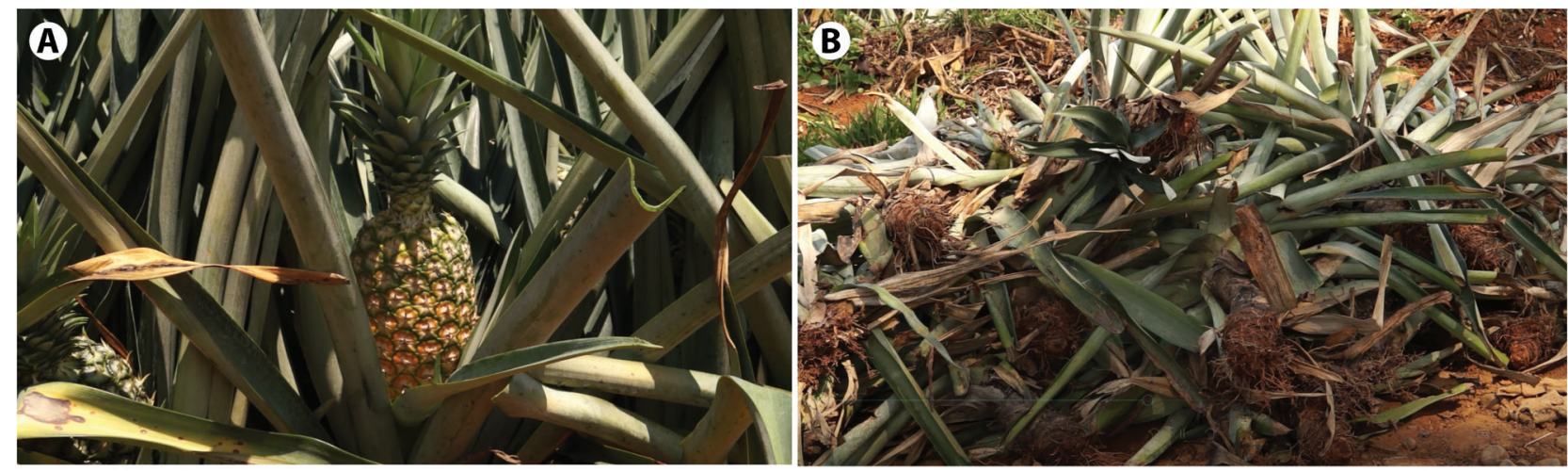

Fig. 3. (A) Fruta de piña con corona e hijos secundarios. (B) Planta de piña como rastrojo (desecho agrícola). Fotografías: R. Hernández.

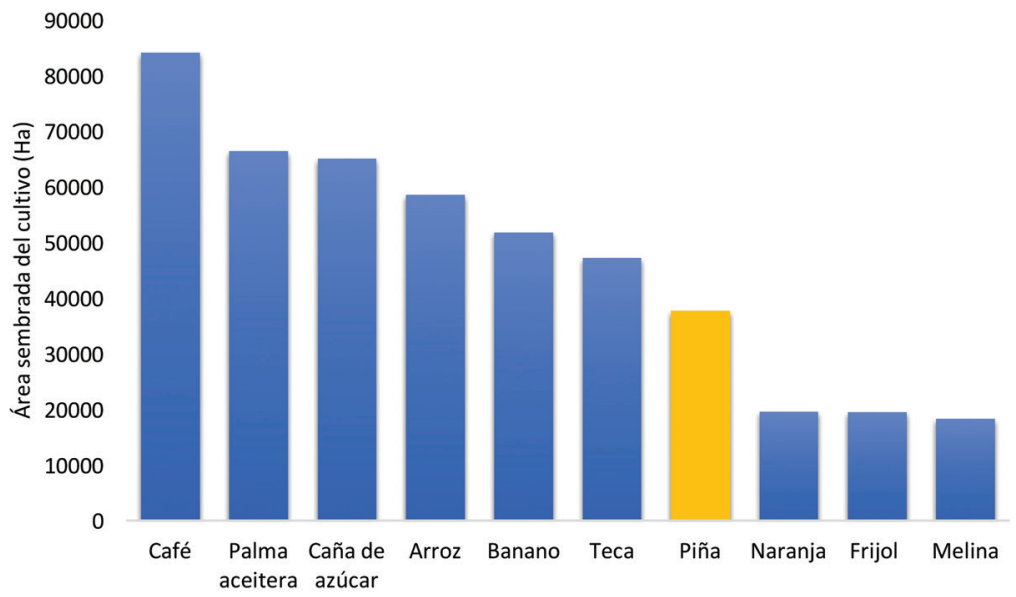

Fig. 4. Área sembrada de los principales cultivos en Costa Rica. Fuente: INEC, 2017.

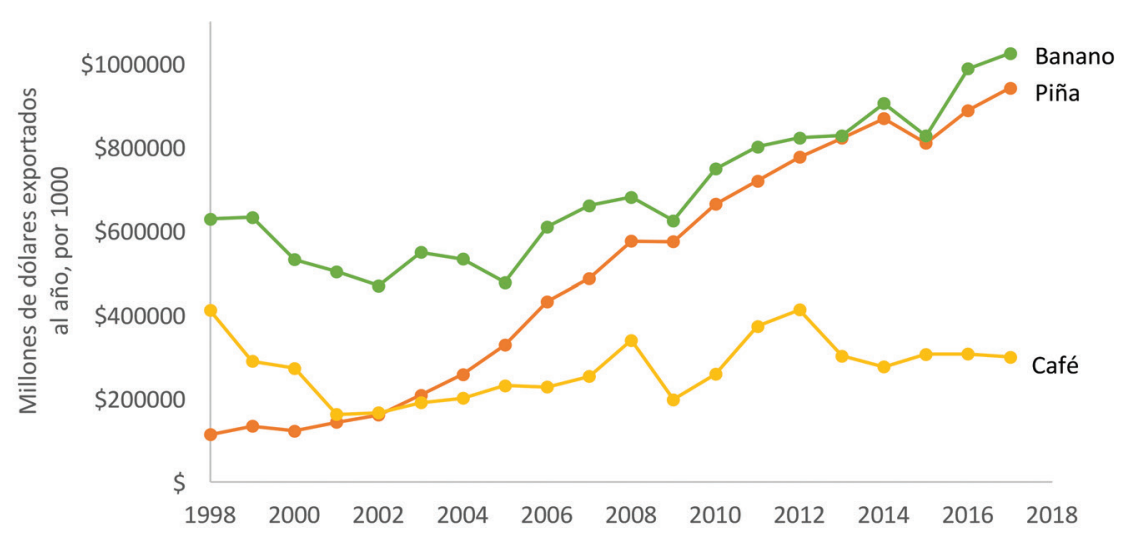

Fig. 5. Exportaciones de los tres principales productos agrícolas de Costa Rica. Fuente: PROCOMER, 2018. 
año). Esto hace que el cultivo de la piña en Costa Rica resalte en importancia, dado su volumen de exportación, en especial cuando se compara con productos agrícolas tradicionales como el café y el banano, los cuales tienen más de 130 años de ser cultivados.

Si se analiza solo el impacto que tiene la actividad de la piña por sus exportaciones de fruta fresca, se observa en la figura 6 , como su crecimiento ha sido constante en los últimos 17 años hasta alcanzar cifras cercanas a los 900 millones de dólares exportados anualmente. En comunicación personal, para García y Valenciano (2017), la inflexión en 2009 se da por efectos climáticos y en parte asociado a la crisis económica mundial, y la de 2014 por factores climáticos y salida de pequeños productores del negocio de la producción. Efectivamente el clima fue determinante en este comportamiento. Lo anterior es reportado por Elizondo (2010), donde indica que los frentes fríos y sistemas de baja presión afectaron a los cultivos de piña. Barquero (2015), en nota periodística citando a Chávez (CANAPEP) y Vargas (PROCOMER), menciona que la baja en las exportaciones se debe a la quiebra de pequeños productores que disminuyó en cerca de $5000 \mathrm{Ha}$ el área sembrada dado por los efectos de la excesiva lluvia. Lo anterior hizo que estos productores se devolvieran al cultivo de raíces, tubérculos, ganadería de doble propósito y pimienta. Es importante considerar, que la piña fresca se exporta con la corona y sin corona; cuando se exporta con corona (follaje de la parte superior no comestible), se paga un valor por una parte que el consumidor no aprovecha.

A partir de comunicaciones personales de Salas (2016), García (2017), Valenciano (2017), Rodríguez (2017) y Miranda (2016), quienes son responsables diferentes de operaciones de producción, calidad y siembra asociada con el cultivo de piña, describen que la actividad tiene la ventaja comercial, qué las frutas que no se exportan como fruta fresca, se procesan en jugos, congelados y pulpas para su exportación y comercio nacional. Ellos igualmente indican que el mercado del jugo es muy volátil en relación al precio de mercado internacional, tal como lo comenta Valenciano en entrevista reciente, donde el precio del jugo esta en uno de sus niveles más bajos. Lo anterior da a la piña una versatilidad de aprovechamiento muy alta y la transformación de esta para su comercialización de diferentes formas. Además, que las solicitudes de mercado son tan amplias, que parte de la fruta se exporta sin la corona como elemento estético y decorativo. A partir de dichas comunicaciones personales, éstos indican que la mayor problemática con el cultivo de la piña para los productores es realizar un manejo integral y responsable del rastrojo de piña, dado el alto volumen de biomasa que se genera. Esto último, se asocia con la densidad de siembra del cultivo, ya que actualmente es cercana a las 73000 plantas por hectárea, e indican que hay ensayos de hasta 85000 plantas por hectárea. Al no realizar un manejo integral, el rastrojo se convierte en foco de contaminación para la propagación de la mosca del establo (Stomoxys calcitrans), ya que es un excelente medio para la incubación de los huevos de la mosca. Esto hace que exista el debate y controversia entre los vecinos de los productores de piña, ya que los catalogan como los responsables por las infestaciones de la mosca y la afectación del ganado vacuno predominante en la zona, y las molestias de la salud pública. Como medida para contrarrestar este problema, los productores colocan trampas para las moscas en las plantaciones y tratan de realizar un proceso eficiente en el manejo del rastrojo. Es importante mencionar que otros cultivos agrícolas generan grandes cantidades de residuos como lo son el café, la palma aceitera, caña de azúcar. Estos dos últimos con la pequeña ventaja que al tener un residuo

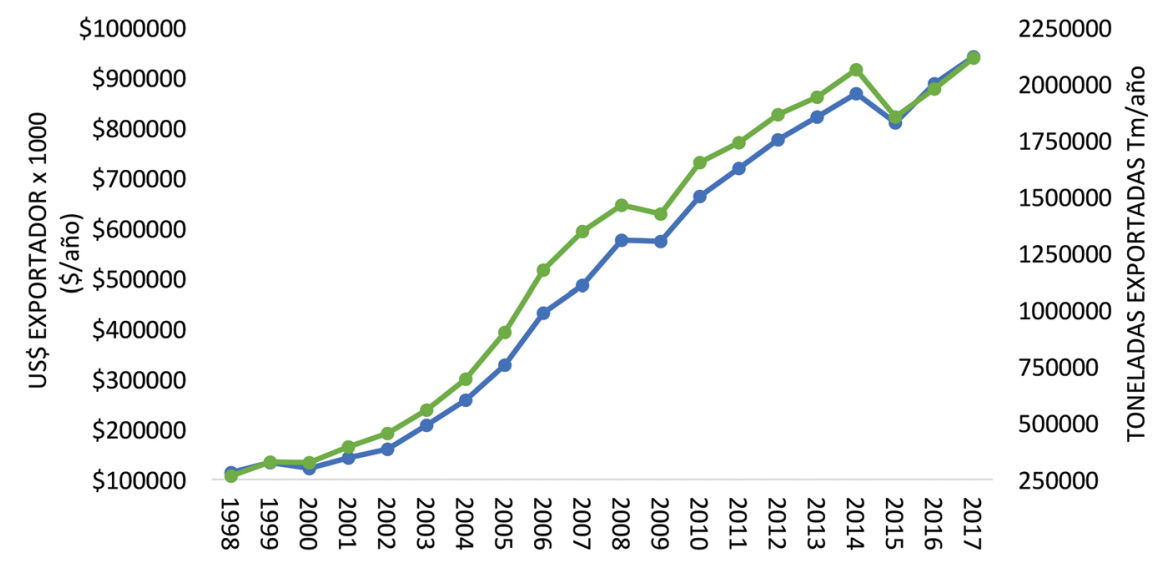

Fig. 6. Exportaciones de piña fresca de Costa Rica. Fuente: PROCOMER (2018). 
de baja humedad en su procesamiento, parte de ellos se utilizan en los sistemas de calderas de los procesos industriales como se puede constatar cuando se realizan vistas de sitio.

Manejo del rastrojo de piña: El rastrojo se genera luego de la primera (si hay efectos climáticos o enfermedades) o segunda cosecha de la planta de piña, muy esporádicamente se llega a un ciclo que cumpla tres cosechas. La primera cosecha se da luego de 14 a 16 meses, mientras que el de segunda entre los 27 y 29 meses, es importante recordar que estas plantas se obtienen los brotes para la resiembra y nuevos ciclos productivos.

Durante observaciones de campo se determinó que el rastrojo se maneja de dos formas, en seco o verde, a continuación, se describe brevemente cada una:

1. El manejo en seco corresponde a la aplicación de herbicida con ingrediente activo (Dicloruro de 1, $1^{\prime}$-dimetil-4,4'-bipiridilo) como medio de secado químico; posteriormente se hace la aplicación de fuego (que$\mathrm{ma}$ ) y los residuos que quedan (algunas hojas, tallos y raíz) se incorporan al terreno por medio de rastras. Esta operación oscila con un costo aproximado entre los $\$ 1000$ y \$1 500 por hectárea, según la topografía y condiciones climatológicas. En la figura 7 se refleja el efecto del manejo en seco.

2. Para el manejo en verde, se elimina la aplicación de herbicida y fuego, pero se mantiene la aplicación de rastras para la molienda e incorporación al terreno, hay una aplicación previa de microorganismos degradadores (en algunos casos). Esta actividad tiene un costo aproximado entre los US\$ 1600 a US\$ 2400 por hectárea. El principal problema a nivel productivo que indican los productores es que el ciclo del uso del terreno se retrasa de tres a cuatro semanas según las condiciones climáticas. En figura 8 se presenta el proceso de secado en verde y como a los alrededores del área tratada ponen trampas para la mosca del establo, los puntos blancos.

La diferencia de costo entre el manejo en seco y verde hace que los productores se decanten por el proceso en seco, ya que el factor de costo es 1.6 veces más caro, estimado a partir de los costos asociados anteriormente. Con la integración de procesos de certificación voluntarios como Rainforest Alliance, los productores buscan de integrar el manejo en verde, dado que una de las condiciones de esta certificación es la eliminación del uso de herbicidas, así como la disponibilidad dentro de las fincas de zonas de protección. Otra forma de manejo, pero que es compleja revisarla o verla en campo, es el de cavar fosas y hacer el entierro del rastrojo en estas fosas, es una práctica que no se realiza regularmente, pero aumenta el fenómeno de lixiviación de sustancias y su incorporación a fuentes subterráneas de agua, como parte de la descomposición de la materia orgánica.

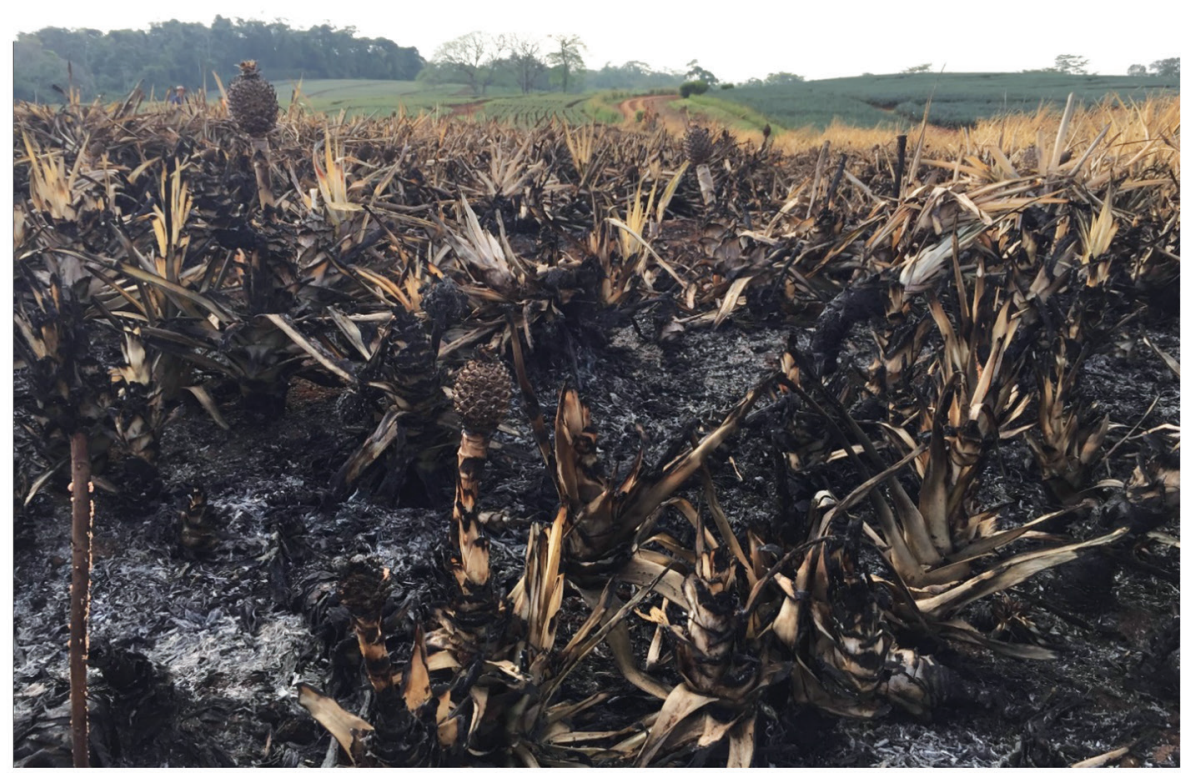

Fig. 7. Efecto del manejo por secado químico y quema en campo. Fotografía: R. Hernández. 


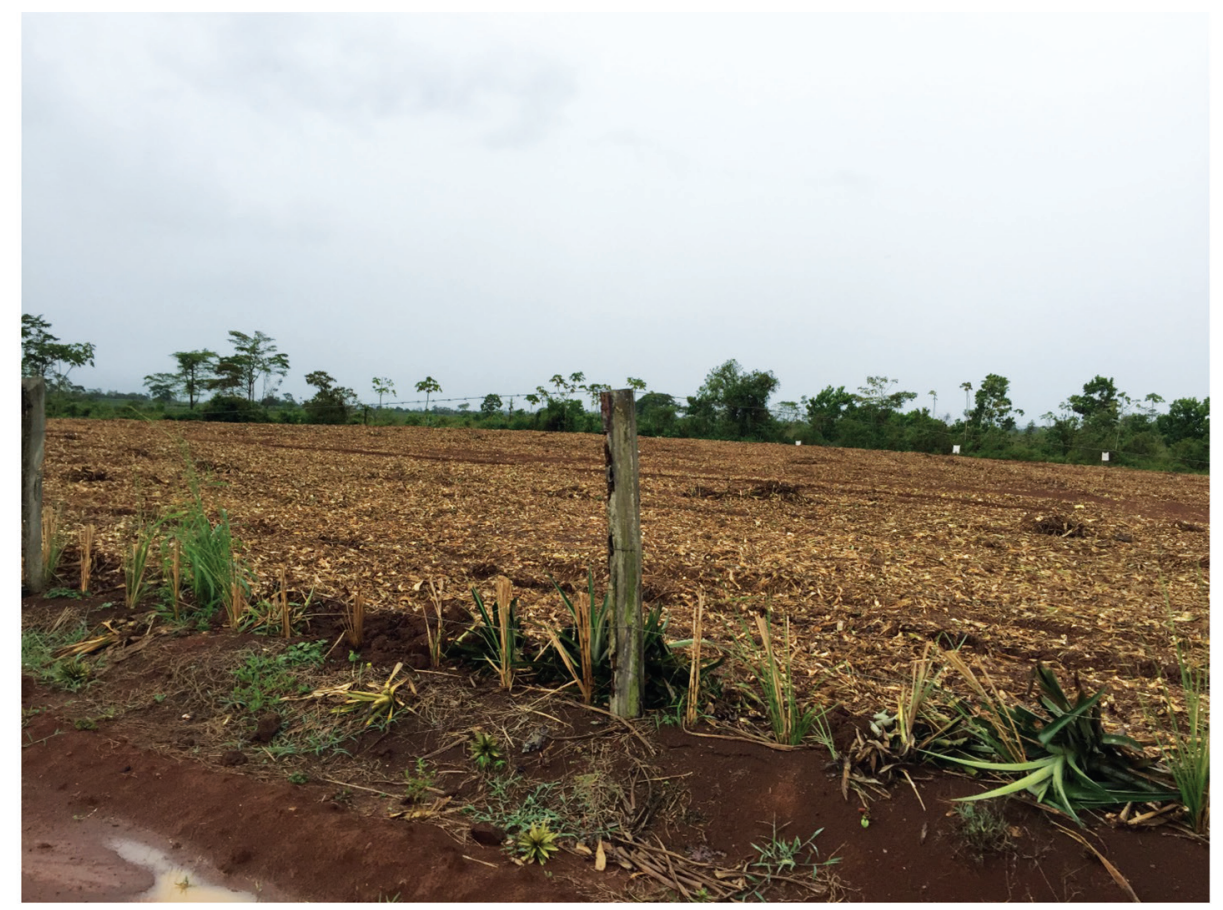

Fig. 8. Manejo en verde del rastrojo. Fotografía: R. Hernández.

Efecto en las áreas de cultivo: A partir de las entrevistas y observaciones de campo, se determinó que la mayor parte del área dedicada al cultivo de piña sufrió un cambio de uso de suelo, donde inicialmente eran suelos dedicados al pastoreo de ganado, cultivos rotatorios como maíz y frijol, naranja, en algunos casos zonas de protección ambiental. Así, al visitar las franjas limítrofes de las áreas de cultivo se observa que éstas son adyacentes con zonas de protección, bosques secundarios y zonas de pastoreo de ganado.

En la figura 9 se observan las áreas de cultivo en una finca de producción de piña, en la misma hay áreas de preparación de terreno, zonas de protección, zonas de cultivo, corredores biológicos o interconexión. Las fincas más responsables manejan aproximadamente una relación de un cincuenta por ciento en área cultivada y área de protección. También se observan zonas, donde las fincas limitan con otros monocultivos como la caña de azúcar.

\section{Posición desde el Sistema Fitosanitario del Estado:} A partir de entrevistas realizadas a personeros del SFE en la zona de estudio, se obtuvo la información necesaria para establecer que la posición del estado está en garantizar que la producción se lleve a cabo en un marco de protección ambiental, normas de salud ocupacional y en el cumplimiento de regulaciones fitosanitarias nacionales e internacionales; dónde se garantice un producto de

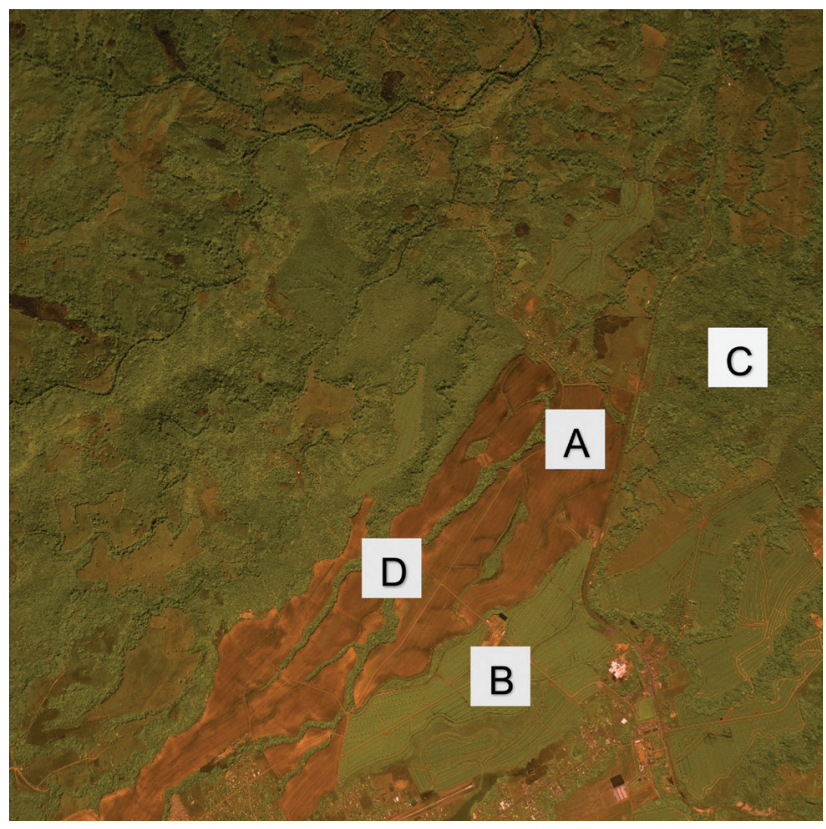

Fig. 9. Fotografía de la Región de Buenos Aires de Puntarenas, Costa Rica. A: Terrero en preparación, B: área cultivada, C: Zona de protección (bosque), D: Corredor de paso (biológico). Fuente. Mosaico Fotogramétrica Proyecto CARTA 2005 CeNAT-Laboratorio PRIAS. 
calidad que permita la competitividad en la conquista de nuevos mercados. Como grupo de trabajo han identificado que los principales problemas que afecta el cultivo de piña en la región desde la visión del SFE son: a. pérdida de suelos, b. altas descargas de agroquímicos en ríos, c. proliferación de plagas, d. desplazamiento de cultivos tradicionales que anteriormente ejercían un papel importante en la seguridad alimentaria, y e. alta demanda de tierras. Además, indican que, con el crecimiento descontrolado de las áreas de cultivo, cada vez se dificulta más las labores de supervisión en el campo y las tomas de muestras para el control fitosanitario.

Es así, que el SFE busca que el cultivo de la piña se lleve a cabo con un manejo integral del rastrojo, que actualmente consiste en realizar una mayor incorporación de la biomasa al suelo sin afectar otras actividades agropecuarias y al cultivo en sí mismo, y que logre:

- La degradación del rastrojo en el menor tiempo posible para evitar proliferación de plagas.

- Mejorar la estructura del suelo, utilizando buenas prácticas agrícolas para conservación de este.

- El uso de sistemas de agricultura de precisión para reducir los daños ambientales.

- El uso de sistemas como mínima labranza y descanso del suelo por largos períodos.

Entre otros elementos que contempla el SFE para el manejo del rastrojo de piña están: a. producción de energía, b. alimento para ganado (ensilado), c. aprovechamiento de la fibra para textiles $\mathrm{u}$ otros.

En relación al compromiso que percibe el SFE sobre la actitud de los productores para mejorar el manejo del rastrojo de piña es baja. Lo anterior se debe a que la actividad principal de ellos es la producción de fruta, sin embargo, existen instituciones y empresas analizando posibles usos de esos desechos. Prueba de ello son algunas acciones que se hacen para aprovechar algunas partes de la planta como las coronas y follaje para la alimentación de ganado (ensilaje) en períodos de crisis por sequía.

Estimación de la biomasa de rastrojo: Los productores indican que la tasa (razón) de recambio anual del cultivo es cercana al $40 \%$ o 0.4 (RRC). La densidad de siembra (DS) es de 73000 plantas por hectárea, una razón de vida de las plantas sembradas del 95\% (RVS). En las mediciones de campo se encontró en esta investigación que la masa media de plantas individuales (MPI) de primera y segunda cosecha es de $3.59 \mathrm{~kg}$, con una desviación estándar de 1.03 kg; aquí García F., (2017) indica que en pruebas de campo han dado seguimiento a medir la masa de la planta en diferentes etapas del crecimiento, y en el momento del descarte como rastrojo han reportado a lo interno de la empresa $3.57 \mathrm{~kg}$, con una desviación estándar de 1.15 kg (único registro encontrado en las fincas y entrevistas). Para un área total de cultivo de 43000 ha (AC), al aplicar la fórmula de la ecuación 1 descrita en la metodología, se obtiene como masa de rastrojo de piña húmedo en base anual.

$$
\begin{aligned}
& \mathrm{MRPH}=4,282 \pm 1,192 \times 10^{6} \text { ton/ } \\
& \text { año en base húmeda (fresco) }
\end{aligned}
$$

Lo anterior es una cantidad considerable de biomasa que queda en el campo y que tiene un alto impacto económico de manejo para el productor y para el ambiente; por lo que es importante establecer estrategias de gestión en la disposición y uso del rastrojo de piña como una materia valorizable. Esta cantidad de biomasa húmeda corresponde a 642300 toneladas anuales en base seca, al considerar una humedad de $85 \%$ establecida en el laboratorio.

Matriz de fortalezas-oportunidades y debilidades-amenazas: Con la información que se ha recopilado y datos obtenidos hasta el momento es factible establecer cuáles son las fortalezas y oportunidades del cultivo de piña, así como sus debilidades y amenazas (Cuadro 1). Este tipo de análisis permite identificar las estrategias básicas para la sostenibilidad del cultivo en el mediano y largo plazo.

Alternativas de uso para el rastrojo de piña: En investigaciones recientes se presentan alternativas de uso para el rastrojo de piña, las mismas han quedado como ensayos a nivel de laboratorio, es importante retomarlas y explorarlas a un nivel de escala piloto de biorrefinería. A partir de este escalamiento a nivel piloto es importante determinar las opciones de procesos a escala comercial para el manejo de los residuos agrícolas del cultivo de piña y otros más que se generan por las actividades de producción de alimentos. En el cuadro 2 se presenta un resumen de éstas investigaciones con rastrojo de piña.

Elementos de un modelo de biorrefinería: Como propone Dimian (2015) la disponibilidad finita de materias primas fósiles, con base en el petróleo o el gas natural, se convierte en un problema crucial para el futuro 
CUADRO 1

Matriz de fortalezas, oportunidades, debilidades y amenazas para el manejo adecuado del cultivo de piña.

\section{Fortalezas}

Altas exportaciones y generación de divisas.

Alto rendimiento por hectárea cultivada.

Aprovechamiento de la cosecha tanto para exportación de fruta fresca como sus derivados.

Fincas productoras con certificaciones internacionales.

\section{Debilidades}

Uso de gran cantidad de agroquímicos.

Mala imagen ante la sociedad al contrastar con la imagen de país amigable con el ambiente.

Hermetismo por parte de los productores para mostrar sus procesos.

Invasión de zonas de protección.

Cambio de uso de suelo.

Alta generación de residuos (rastrojo de piña).

Cantidad de agroquímicos utilizados y su falta de cuantificación.

Vulnerabilidad del cultivo a efectos climáticos.

Falta de experiencia e investigación en el área de la biorrefinería a gran escala en el país.

\section{Oportunidades}

\section{Amenazas}

Aprovechamiento de la biomasa en procesos de biorrefinería Uso de la biomasa para ensilaje.

Pérdida de imagen de país comprometido con el ambiente.

Pérdida de mercado

Mejor imagen del cultivo ante sociedad al usar mejores técnicas de manejo de residuos.

Expansión del cultivo en otros países del área.

Que otros países usen técnicas de manejo de residuos del cultivo de piña según estándares de certificación y que perjudiquen las

Integración con las comunidades vecinas al cultivo para el emprendimiento de micro negocios con el residuo y dar valor agregado.

Obligatoriedad de normativas internacionales nuevas.

\section{Estrategias vinculantes}

Controlar la expansión del cultivo de piña sin afectar zonas de protección, patrimonio o cambios drásticos de uso de suelo.

Caracterizar la biomasa residual del cultivo de piña (rastrojo) para establecer opciones de valorización.

Establecer una estrategia país para el manejo de los residuos biomásicos desde una perspectiva de proceso de biorrefinería.

Trabajar de forma colaborativa productores-instituciones de gobierno-universidades para desarrollar investigación a mediano y largo plazo para el uso de la biomasa residual.

de las industrias de procesos químicos, por lo que el uso de materias primas renovables, como la biomasa entraría en la ecuación de su sustitución a mediano y largo plazo. Por lo tanto, se deben plantear este tipo de alternativas y el uso de los residuos agroindustriales para la obtención de sustancias con valor agregado.

Quintero Bertel, Silva Lora y Melian Cobas (2014), indican que es importante diseñar un sistema agro-energético para la gestión de los recursos de manera sostenible. Este sistema debe incluir aspectos técnicos, económicos, sociales y ambientales articulados con políticas públicas de gestión de acuerdo con las necesidades de desarrollo de cada país, haciendo control de los procesos mediante indicadores adecuados, y así garantizar costos accesibles de la energía con bajas emisiones de $\mathrm{CO}_{2}$, generación de empleos y servicios ambientales.

A partir del modelo de Quintero-Bertel, Costa Rica y otros países comprendidos en la zona intertropical, están en un momento crucial para implementar un modelo en el manejo de la biomasa lignocelulósica proveniente del rastrojo de piña, y de otras biomasas provenientes de su modelo agrícola (caña de azúcar, banano, café, ganadería, entre otras). El sistema agro-energético debe tener como base un proceso de biorrefinería articulado en escalas, con el objetivo de minimizar los costos logísticos de transporte y promover el autoconsumo. Actualmente se utiliza el bagazo de caña en autoconsumo para la producción de energía en los ingenios, se hace el esfuerzo 
CUADRO 2

Investigaciones con rastrojo de piña o residuos de piña.

\begin{tabular}{|c|c|c|}
\hline Estudio & Observaciones & Referencia \\
\hline $\begin{array}{l}\text { MTBE y calor de } \\
\text { combustión }\end{array}$ & $\begin{array}{l}\text { Calor de combustión de la corona de piña de } 17,1 \pm 0,5 \mathrm{MJ} / \mathrm{kg} \text { y } \\
\text { de } 16,1 \pm 0,3 \mathrm{MJ} / \mathrm{kg} \text { para el rastrojo. La conversión química para el } \\
\text { sustituto del MTBE con un rendimiento máximo del } 47,75 \pm 0,01 \% \text {, } \\
\text { de una mezcla de compuestos furánicos (mayoritariamente } \\
\text { 5-(hidroximetil)furfural y levulinato de metilo) }\end{array}$ & Irías-Mata \& Lutz (2013) \\
\hline Bioetanol & Concentración de etanol promedio obtenida fue de 1,87\% v/v & Córdoba \& Molina (2014) \\
\hline Biogas & $\begin{array}{l}\text { Producción de biogás de } 25,7 \text { litros de metano por cada kilogramo } \\
\text { de rastrojo de piña fresco }\left(171,3 \mathrm{~mL} \mathrm{CH}_{4} / \mathrm{gr} \mathrm{SV}\right)\end{array}$ & Arce, Hernández \& Amador (2014) \\
\hline Bioetanol & $\begin{array}{l}\text { Se obtuvo bioetanol mediante destilación, presentando un } \\
\text { rendimiento del } 35 \% \text { con bagazo de piña y del } 57 \% \text { con celulosa } \\
\text { con un tiempo de fermentación de } 48 \text { y } 72 \text { h, respectivamente }\end{array}$ & Cruz et al. (2011) \\
\hline Bioetanol & $\begin{array}{l}\text { Concentración de bioetanol de } 7.12 \% \text { (v/v) por medio de } \\
\text { fermentación en medio sólido. }\end{array}$ & Chintagunta, Ray \& Banerjee (2017) \\
\hline Papel & $\begin{array}{l}\text { Producción de papel a partir de las hojas de las plantas de piña de } \\
\text { desecho, mezclando fibras de caña y papel de desecho. }\end{array}$ & Sibaly \& Jeetah (2017) \\
\hline Bioetanol y bromelina & $\begin{array}{l}\text { Producción de bioetanol utilizando sacarificación y fermentación } \\
\text { simultaneas, y extracción de bromelina. Resultado de bioetanol }(5,4 \\
\%(\mathrm{v} / \mathrm{v}) \text { y bromelina } 11,5 \text { a } 21,0 \mathrm{mg} / \mathrm{mL} \text {. }\end{array}$ & Seguí \& Maupoey (2018) \\
\hline
\end{tabular}

de producir biogás con los desechos de la industria del café, y lo propio con los residuos de la palma aceitera. Por lo cual el modelo a seguir con el rastrojo de piña y otras biomasas debe inicialmente sustentarse en los siguientes pasos:

1. Caracterización fisicoquímica del rastrojo de piña y otras biomasas.

2. Evaluar qué rutas o combinaciones de conversión de materiales se pueden establecer (térmica, bioquímica, biológica).

3. Establecer alianzas de cooperación sector privado-gobierno-universidad.

4. Evaluar la integración de diferentes tipos de biomasas lignocelulósicas con potencial bioenergético o para obtención de bioproductos de alto valor agregado.

5. Identificar tierras agotadas para recuperación de las mismas con el cultivo de especies bioenergéticos.

6. Trabajar sobre modelos de planta piloto para escalar a nivel industrial.

7. Realizar simulaciones de ciclo de vida para establecer impactos de las propuestas sobre el ambiente, sociedad y economía local.

8. Realizar análisis de mercados con otros países (Estados Unidos, Alemania, España, etc) con mayor experiencia en procesos de biorrefinería para ajustar a las condiciones de la región y sus biomasas.
9. Articular una ruta país y la legislación necesaria para promover el uso de biomasas en la producción energética y obtención de productos de alto valor agregado.

Esto nos lleva a que el uso de biomasas específicas, de residuos de la agroindustria o de las actividades humanas en procesos de biorrefinería es una forma de minimizar los impactos de las acciones antropocéntricas sobre el planeta. Lo anterior es una ventana para ayudar en el cumplimiento de los objetivos de desarrollo sostenible como seguridad alimentaria, consumo y producción responsable, acción por el clima y producción de energía sostenible, entre otros. Un modelo que se propone es el que se muestra en la figura 10, donde se debe tomar como una estrategia país o regional los procesos de biorrefinería en búsqueda de un macro modelo de economía circular o economía verde. Donde a partir actividades socio-ambiental-económicas se debe desarrollar la producción de cada país, para maximizar la generación de productos y minimizar el impacto sobre el ambiente, en beneficio del mayor número de individuos de la sociedad. Así, en la figura 10 se parte de los residuos de las actividades agrícolas, agroindustriales y humanas para establecer un modelo de biorrefineria, donde se deben caracterizar las biomasas, identificar la geografía para el transporte y flujo de las mismas al centro de proceso. Una vez en el centro de proceso realizar un análisis de ciclo de vida del producto e identificar si es viable establecer un 


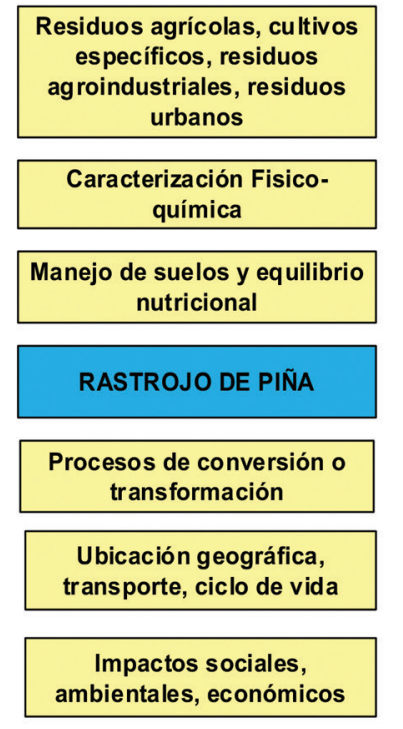

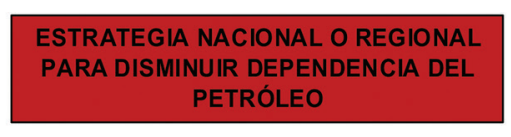
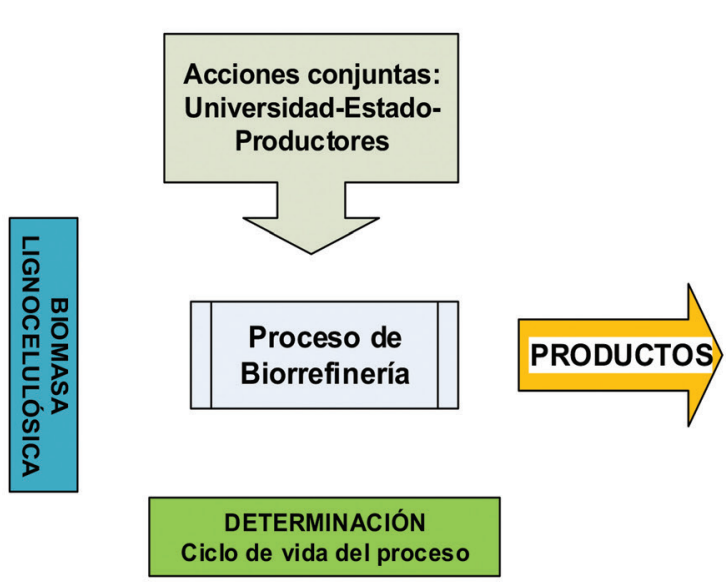

Biocombustibles líquidos, sólidos, gaseosos
Nuevos materiales para la manufactura o síntesis industrial

Residuos del proceso y su análisis para valorización

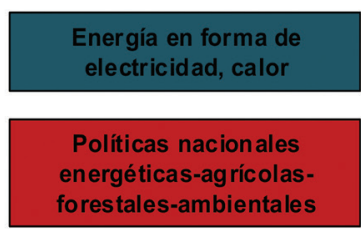

Fig. 10. Propuesta de modelo de biorrefinería.

modelo de ecología industrial alrededor de la biorrefinería para disminuir la generación de residuos. Todo este esfuerzo debe tener un impacto mayor, ya que al juntar a productores-gobierno-universidad, se deben establecer políticas nacionales en materia energética, ambiental, forestal y agrícolas para consolidar el modelo y el progreso del país y sus ciudadanos.

Rastrojo de piña y un modelo de biorrefinería: Es aquí, donde nuestra propuesta de un modelo de biorrefinería alrededor inicialmente del rastrojo de piña toma vida de manera conceptual. La misma implica tomar el rastrojo de piña (causa) y transformarlo en sustancias de valor agregado bajo un modelo de biorrefinería (efecto). En figura 11 se expone el modelo gráfico potencial.

Se observa como el rastrojo entra al proceso, una aplicación inicial para cubrir demanda energética es el secado al sol para la combustión del mismo. Se pasa de realizar una combustión actual según el tratamiento de secado químico, donde se desperdicia esa energía y se generan gases de efecto invernadero, a tener un uso energético base. Otra fracción del rastrojo se lleva a procesos de producción de biogás y abono, donde por medio de la acción microbiana anaerobia se puede producir un combustible como el biogás y un abono para los suelos. Por medio de un proceso de fermentación se pueden producir alcoholes como el etanol o butanol, con aplicación bioenergética o como sustancias de síntesis para la producción de otras. Según las condiciones del proceso de pueden obtener sustancias como el hidroximetilfurfural o el furfural como intermediarios de síntesis más complejas para productos de mayor valor agregado. También, existe la posibilidad de integrar la recuperación de fibra natural del rastrojo (especifico de las hojas) para la producción de cuerdas o su uso como material de refuerzo.

Es importante indicar que se deben realizar todos estudios sobre impacto, huella de carbono y ciclo de vida

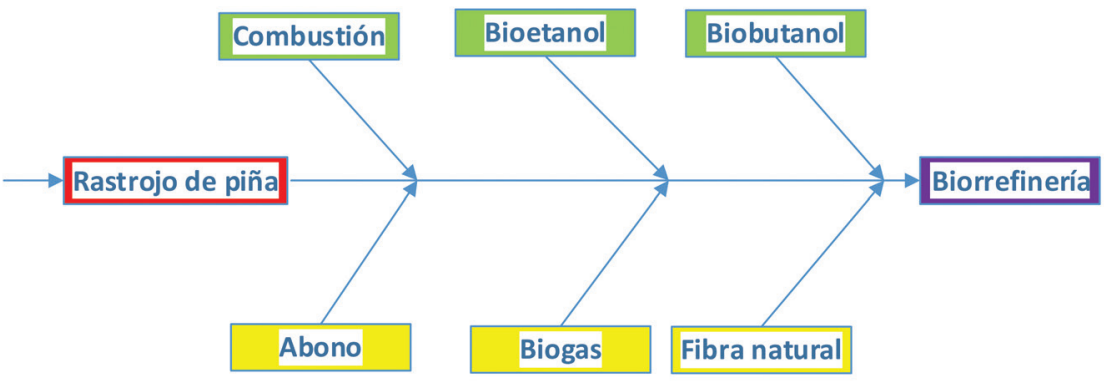

Fig. 11. Modelo de biorrefinería para el rastrojo de piña. 
de la variedad de productos que se obtengan desde un proceso de biorrefinería. Lo anterior considerando las distancias de transporte de la materia prima (rastrojo de piña), forma de cosecharlo en las fincas, donde realizar una primera reducción de tamaño, etc. Elementos importantes a considerar en su cosecha es que no se haya aplicado herbicidas para su secado, así como, la disminución de tamaño en campo para aumentar la masa transportada por unidad de viaje realizado.

Lo anterior nos deja en la antesala de procesos investigativos vinculantes con el potencial del rastrojo de piña como una materia prima para un aprovechamiento en procesos de biorrefinería, donde se maximice su uso y se minimice la generación de residuos bajo un modelo de bioeconomía, además, tendría un posible efecto potenciador en zonas afectadas por el monocultivo, para que se generen encadenamientos productivos asociados con la cosecha, transporte, procesamiento del rastrojo antes de llegar la planta procesadora final, permitiendo dinamizar la economía a pequeña escala de estas zonas.

El uso de biomasas específicas o no, como el rastrojo de piña en la producción bioenergética y de sustancias de valor agregado, tendrá en el mediano y largo plazo impactos positivos en el manejo de los residuos agroindustriales, ayudando a las economías locales y al medio ambiente. Esto dependerá de las interacciones de trabajo colaborativo que se generen entre las diferentes partes como universidad-gobierno-industria, que demarquen rutas en cada país en cumplimiento de las expectativas de carbono neutralidad en los próximos años y los objetivos de desarrollo sostenible con metas concretas al año 2030.

\section{AGRADECIMIENTOS}

Agradecemos al Sistema Fitosanitario del Estado, agencia de Ciudad Quesada. Francisco García, Hacienda la Lidia. Alexandra Miranda, Fyffes Costa Rica. Stephanie Rodríguez, CANAPEP. Luis Salas, Tico Fruit S.A. Luis Valenciano, Agrovicceses y Monte de la Providencia. Julio Mata-Segreda, Universidad de Costa Rica. Proyecto AMIUNED por el financiamiento a proyecto de Doctorado.

\section{REFERENCIAS}

Arce, A., Hernández, C., \& Amador, R. (2014). Determinación de la cantidad y composición de biogás a partir del rastrojo de piña (ananas comosus) por medio de un sistema continuo de laboratorio. San José: ICE.
Barquero, M. (2015, 12 de setiembre). Exportación de piña cae por mal clima y salida de productores. La Nación, Costa Rica.

CANAPEP. (2017). Gráfico de distribución del área sembrada. Recuperado de http://canapep.com/

Chintagunta, A.D., Ray, S., \& Banerjee, R. (2017). An integrated bioprocess for bioetanol and biomanure production from pineapple leaf waste. Journal of Cleaner Production, 165(2017), 1508-1516.

Córdoba, M., \& Molina, M. (2014). Determinación del efecto de la concentración de la celulasa, celobiasa y de $\mathrm{NaOH}$ en la hidrólisis para la producción de etanol a partir de rastrojo de la piña. Revista Ingeniería UCR, 24(2), 19-25.

Cruz, R., Mendoza, M., Chávez, Y., Rivera, L., \& Cruz, J. (2011). Aprovechamiento del bagazo de piña para obtener ceIulosa y bioetanol. Afinidad LXVIII, 551, Enero - Febrero.

De Pablo Valenciano, J., Tassile V., \& Giacinti Battistuzzi, M.A. (2017). Cambios en el modelo del comercio internacional de piña en Costa Rica. Agroalimentaria, 23(44), 45-60.

Dimian, A (2015). Biorefinery the future of chemical process industries. The Bulletin of the Romanian Chemical Society, 22(1), 15-44.

Elizondo, A. (2010). Análisis del mercado de piña. Servicio de Información e Inteligencia de Mercados, Consejo Nacional de la Producción, 15(1), junio 2010.

García, F. (5 de febrero de 2017). Gerente de producción agrícola Hacienda la Lidia. (R. Hernández, Entrevistador)

Garita Coto, R. A. (2014). La Piña. Cartago Costa Rica: Editorial Tecnológica de Costa Rica,

INEC (Instituto Nacional de Estadística y Censos). (2017). VI Censo Nacional Agropecuario de 2014. Recuperado de http://www.inec.go.cr/sites/default/files/documetos-biblioteca-virtual/01._atlas_estadistico_agropecuario_2014.pdf

Irías-Mata, A.,\& Lutz, G. (2013) Pineapple-stover derived furan compounds as gasoline oxygenate additive. UNED Research Journal, 5(2), 279-282.

Maglianesi Sandoz, M. A. (2013) Desarrollo de las piñeras en Costa Rica y sus impactos sobre ecosistemas naturales y agro-urbanos. Biocenosis, 27 (1-2), 62-70.

Miranda, A. (7 de agosto de 2016) Gerente de Calidad de Fyffes Costa Rica. (R. Hernández Chaverri, Entrevistador)

Navarro, P. J., \& Gómez, L. A. (2015) Diversidad de mamíferos terrestres en bosques cercanos al cultivo de piña, Cutris de San Carlos, Costa Rica. UNED Research Journal, 7(1), 59-65.

PROCOMER. (2018). Sistema estadístico de la Promotora de Comercio Exterior. Recuperado de http://sistemas.procomer.go.cr/estadisticas/inicio.aspx 
Programa Estado de la Nación. (2015). XXI Informe Estado de la Nación en Desarrollo Humano Sostenible. San José: Programa Estado de la Nación.

Quesada-Solís, P., Alvarado Aguilar, P., Sibaja Ballestero, R., \& Vega Baudrit, J. (2005) Utilización de las fibras del rastrojo de piña (Ananas comosus, variedad champaka) como material de refuerzo en resinas de poliéster. Revista Iberoamericana de Polímeros, 6(2), 157-179.

Quintero Bertel, Q. R., Silva Lora, E. E., \& Melian Cobas, V. (2014). Cultivos energéticos para la generación de energía eléctrica. In J. M. Rincón Martínez, E. E. Silva Lora, J. M. Rincón Marínez, \& E. E. Silva Lora (Eds.), Bioenergía: Fuentes, conversión y sustentabilidad (pp. 57-88). Bogotá: Charlie's Impresores Ltda.

Rodríguez, S. (3 de junio de 2016). Coordinadora de Responsabilidad Social y Ambiental CANAPEP. (R. Hernandez Chaverri, Entrevistador)
Salas, L. (10 de mayo de 2016). Gerente de Producción Tico Fruit S.A. (R. Hernández Chaverri, Entrevistador)

Salazar, O. (2008). Plantaciones de piña en Costa Rica contra la sostenibilidad ecológica y social. Ambientico, 177, 13-14.

Seguí Gil, P., \& Fito Maupoey, P. (2018). An integrated approach for pineapple waste valorisatio. Bioethanol production and bromelain extraction from pineapple residues. Journal of Cleaner Production, 172 (2018), 1224-1231

Sibaly, S., \& Jeetah P. (2017) Production of paper from pineapple leaves. Journal of Environmental Chemical Engineering, 5, 5978-5986.

Sistema Fitosanitario del Estado. (5 de abril de 2017). Agencia de SFE de Ciudad Quesada. R. Hernández Chaverri, Entrevistador)

Valenciano, L. (5 de febrero de 2017). Gerente de Calidad Agrovicceses y Monte de la Providencia. (R. -Hernández Chaverri, Entrevistador) 